\title{
Circulating Antibodies to Linear Peptide Antigens Derived from ANXA1 and FOXP3 in Lung Cancer
}

\author{
WEILI WANG ${ }^{1}$, WEN ZHONG ${ }^{2}$, CAIREN CHEN ${ }^{3}$, QINGYONG MENG ${ }^{3}$ and JUN WEI ${ }^{4}$ \\ ${ }^{I}$ Department of Radiation Oncology, Indiana University School of Medicine, Indianapolis, IN, U.S.A.; \\ ${ }^{2}$ Department of Radiation Oncology, the Fourth Affiliated Hospital, \\ China Medical University, Shenyang, P.R. China; \\ ${ }^{3}$ School of Clinical Laboratory Science, Guangdong Medical College, Dongguan, P.R. China; \\ ${ }^{4}$ Department of Division of Health Research, University of the Highlands \& Islands, \\ Centre for Health Science, Inverness, U.K.
}

\begin{abstract}
Background: Our previous studies revealed that concentrations of circulating antibodies to annexin Al (ANXA1) and forkhead-box P3 (FOXP3) increased significantly in patients with non-small cell lung cancer (NSCLC). This study was thus undertaken to replicate our initial findings with different sample sets. Patients and Methods: Antibodies were tested in 108 patients with NSCLC and 216 controls, who were divided into the discovery $(49$ vs. 108) and validation (60 vs. 108) group based on the time of enrolment. Results: Analysis of the discovery group showed a significant increase in circulating anti-ANXA1 IgG levels in the patient group compared with the control group $(p=0.005)$ but the validation group simply exhibited a trend toward an increase in $\operatorname{IgG}$ levels in NSCLC $(p=0.238)$, generating a combined p-value of 0.009. Conclusion: The findings of this study support the notion that circulating $\operatorname{Ig} G$ antibodies to ANXA1 could be used as a biomarker for early diagnosis of NSCLC but failed to replicate such findings for FOXP3.
\end{abstract}

Circulating antibodies to particular tumor-associated antigens (TAAs) have been found to be positive in a certain group of patients with malignant tumors (1-5), although the TAAs involved in specific immune responses differ between tumor types and between individuals with the same tumor type. Spontaneous tumor-related antibodies have also been considered as biomarkers for early diagnosis and prognosis

Correspondence to: Weili Wang, MD, Ph.D., Department of Radiation Oncology, Indiana University School of Medicine, 535 Barnhill Drive, Indianapolis, IN 46202, U.S.A. Tel: +1 3172747305 , e-mail:ww32@iu.edu

Key Words: Tumor-associated antigens, ANXA1, FOXP3, antibody test, non-small cell lung cancer. of malignancies $(2,5-11)$. The EarlyCDT ${ }^{\circledR}$-Lung test was the first autoantibody-based diagnostic tool in lung cancer (1214), which was made with a panel of seven TAAs: p53, NYESO-1, CAGE (also called DEAD box protein 53, DDX53), GBU4-5, HuD, melanoma antigen gene-A4 (MAGE A4) and sex determining region Y-box 2 (SOX2). While these seven TAAs achieved a panel sensitivity of $47 \%$ against a specificity of $90 \%$ (13), the positivity in patients with stage I non-small cell lung cancer (NSCLC) remained under $30 \%$. It is thus important to identify more TAAs that are suitable for the development of early diagnostic tools for lung cancer.

Recently, we developed a simple enzyme-linked immunosorbent assay (ELISA) with linear peptide antigens for detection of circulating antibodies to TAAs in lung cancer (15-17). The costs for synthetic peptide antigens are much lower than those for recombinant proteins used to develop ELISA antibody tests by most commercial companies. Therefore, ELISA developed with linear peptide antigens may promising in future application. In recent studies, we found that concentrations of circulating antibodies to synthetic linear peptides derived from annexin A1 (ANXA1) and forkhead box P3 (FOXP3) were significantly higher in patients with NSCLC than healthy controls $(18,19)$. In order to confirm whether the results using ELISA developed with linear peptides is reproducible, the present work applied an in-house ELISA with linear peptides derived from ANXA1 and FOXP3 to replicate the initial finding in a different collection of samples from patients with NSCLC.

\section{Patients and Methods}

Patients and controls. A total of 109 patients newly diagnosed as having NSCLC were recruited by the Fourth Affiliated Hospital of the China Medical University, Shenyang, China, in the period between March 2013 and November 2014. The patients had a mean age of $62.1 \pm 10.4$ years, 70 were male and 39 were female. The 109 
Table I. Information on peptide antigens used for the development of enzyme-linked immunosorbent assay antibody test.

\begin{tabular}{lccc}
\hline Antigen & Sequence $(\mathrm{N}-->\mathrm{C})$ & NCBI accession & Working solution $(\mu \mathrm{g} / \mathrm{ml})$ \\
\hline ANXA1 & H-fntilttrsypqlrrvfqkytlirimvsrseid-OH & NP_000691 & 10 \\
FOXP3 & H-iyhwftrmfaffrnhpatwknairhnlslhkd-OH & NP_001107849 & 10 \\
Control & H-haqlegrlhdlpgcprevqrgfaatlvtn-OH & 1BFA_A & 10 \\
\hline
\end{tabular}

ANXA1: Annexin A1; FOXP3: forkhead-box P3.

patients were divided into a discovery group $(n=49)$, with samples that were collected during 2013 , and a validation group $(n=60)$, with samples that were collected during 2014. Their diagnosis and tumor staging were made based on radiographic examination and histological confirmation; inclusion of patients was restricted to those with adenocarcinoma and squamous cell carcinoma only. Blood samples were taken prior to any anticancer treatment. A total of 216 healthy individuals, well matched in age $(59.1 \pm 3.5$ years $)$ and smoking history, were also recruited from local communities, 108 of whom were used as controls for the discovery samples and 108 for the validation samples. Clinical interview and radiographic examination were applied to rule out controls who had a history of lung cancer or any other malignant tumor. All the participants were of Chinese Han origin and they all gave their written informed consent to participate in this study as approved by the Ethics Committee of the Fourth Affiliated Hospital of China Medical University (\#CMU4H2014026), which conformed with the requirements of the Declaration of Helsinki.

Autoantibody testing. To detect circulating IgG to ANXA1 and FOXP3, an in-house ELISA was developed using linear peptide antigens derived from ANXA1 and FOXP3, namely human antigens (hAgs), based on our previous method with modification(18, 19); a peptide fragment derived from a maize protein (NCBI accession 1BFA_A) was used as the control antigen (cAg). The sequence information of these three peptides is given in Table I. Briefly, both hAgs and cAg were synthesized by solid-phase chemistry with purity of $>95 \%$, and then applied to develop a relative ELISA test for detection of circulating IgG antibodies to ANXA1 and FOXP3. Synthetic peptides were dissolved in $67 \%$ acetic acid to obtain a concentration of $5 \mathrm{mg} / \mathrm{ml}$ (stock solution kept at $-20^{\circ} \mathrm{C}$ ), and were diluted with phosphate-buffered saline (PBS)-based coating buffer (P4417; Sigma-Aldrich, St. Louis, MO, USA) containing $0.1 \%$ sodium azide. Coaster 96-Well Microtiter EIA Plates (ImmunoChemistry Technologies, Bloomington, MN, USA) were half-coated in $0.1 \mathrm{ml} /$ well of each $\mathrm{hAg}$ and half-coated in $0.1 \mathrm{ml} /$ well of cAg. The antigen-coated 96-well microplate was incubated overnight at $4^{\circ} \mathrm{C}$. After the plate was washed three times with wash buffer made from Tris-buffered saline with Tween ${ }^{\circledR 2} 20$ (T9039; Sigma-Aldrich), $100 \mu \mathrm{l}$ of plasma sample diluted 1:150 in assay buffer (PBS containing 1.5\% Bovine Serum Albumin) was added and $100 \mu \mathrm{l}$ assay buffer was also added to the negative control (NC) wells. Following 2-hour incubation at room temperature, the plate was washed three times and $100 \mu$ peroxidase-conjugated goat antibody to human IgG (A8667; Sigma-Aldrich) diluted 1:30000 in assay buffer was added to each well. After incubation at room temperature for 1 hour, color development was initiated by adding $100 \mu$ l Stabilized Chromogen (SB02; Life Technologies, Camarillo,
CA, USA) and terminated $25 \mathrm{~min}$ later by adding $50 \mu \mathrm{l}$ Stop Solution (SS04; Life Technologies). The measurement of optical density (OD) was completed on a microplate reader within $10 \mathrm{~min}$ at $450 \mathrm{~nm}$ with a reference wavelength of $620 \mathrm{~nm}$.

Each sample was tested in duplicate. In order to reduce the interference from non-specific signal produced by passive absorption of various IgG antibodies in plasma to the surface of 96well microplate, a specific binding index (SBI) was used to express the levels of circulating antibodies to ANXA1 and FOXP3.

SBI was calculated as follows:

$$
\mathrm{SBI}=\left(\mathrm{OD}_{\mathrm{hAg}}-\mathrm{OD}_{\mathrm{NC}}\right) /\left(\mathrm{OD}_{\mathrm{cAg}}-\mathrm{OD}_{\mathrm{NC}}\right)
$$

Statistical analysis. Antibody-testing data are expressed as the mean \pm standard deviation (SD) of SBI. Student's $t$-test was applied to examine the difference in SBI between the patient group and the control group, and the Fisher's method of combining $p$-values as introduced by Elston (20) was applied to calculate combined $p$ values based on two $t$-tests performed on the discovery samples and the validation samples. Two-tailed tests were performed for statistical significance at a level of $p<0.05$. All statistical analyses were performed using IBM ${ }^{\circledR}$ SPSS Statistics 22.0 (IBM Corp., Armonk, NY, USA). Scatter plot figures of SBI in different groups were generated using GraphPad Prism ${ }^{\circledR}$ version 5.01 (GraphPad Software, Inc., La Jolla, CA, USA).

In order to minimize intra-assay deviation, the ratio of the difference between duplicated OD values to their sum was used to assess the precision for assay of each sample. When the ratio was found to be $>15 \%$, the test of this sample was treated as being invalid and was not used for data analysis. The inter-assay deviation was estimated using a pooled plasma sample, namely a quality control sample, which was randomly collected from $>100$ unrelated healthy individuals.

\section{Results}

Analysis of the discovery samples showed a significant increase in circulating anti-ANXA1 IgG levels in patients with NSCLC compared with controls $(p=0.005)$, while this alteration failed to be replicated in the validation samples $(p=0.238)$, generating a combined $\mathrm{p}$-value of 0.009 (chisquare $=13.47, \mathrm{df}=4$ ) (Table II and Figure 1A).

Neither the discovery samples nor the validation samples showed a significant increase in circulating anti-FOXP3 IgG levels in patients with NSCLC compared with controls; the combined $p$-value was 0.093 (chi-square $=7.97, \mathrm{df}=4$ ) (Table II and Figure 1B). 
A

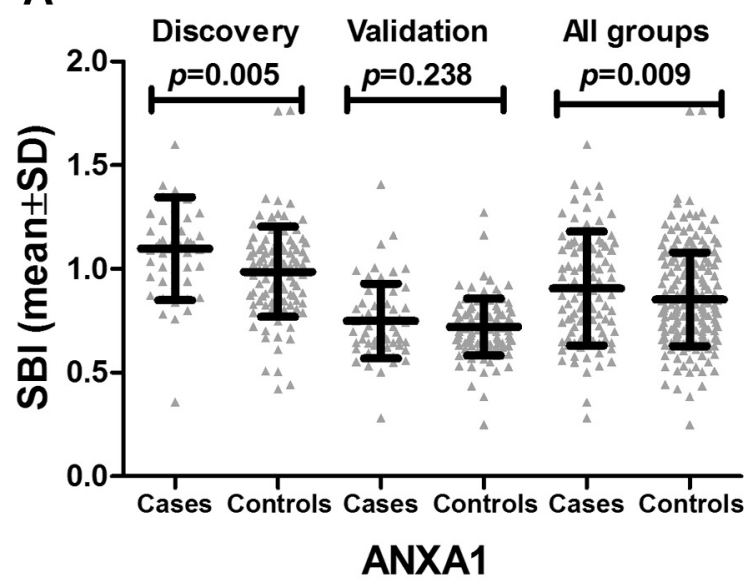

B

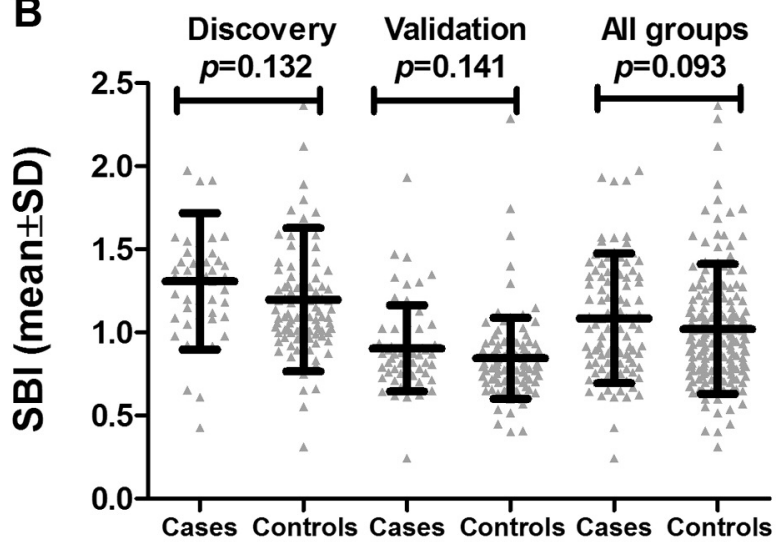

FOXP3

Figure 1. The mean differences and standard deviations of circulating IgG antibodies to annexin A1 (ANXA1) (A) and forkhead-box P3 (FOXP3) (B) between lung cancer cases and healthy controls. p-Values shown are from independent t-tests. SBI: Specific binding index.

A

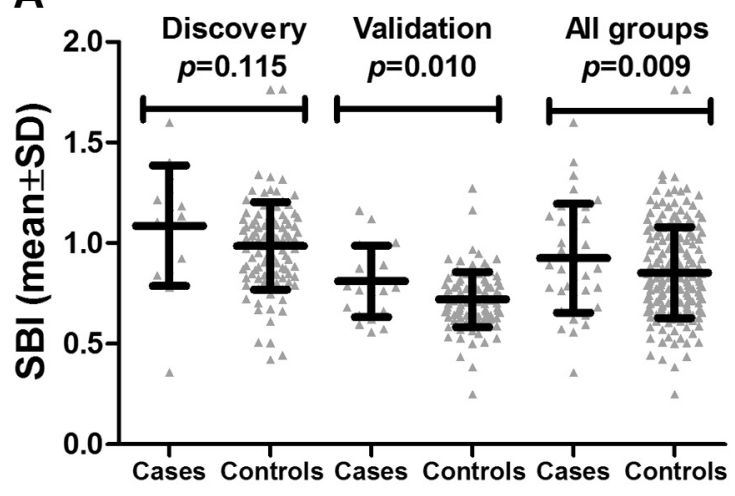

ANXA1

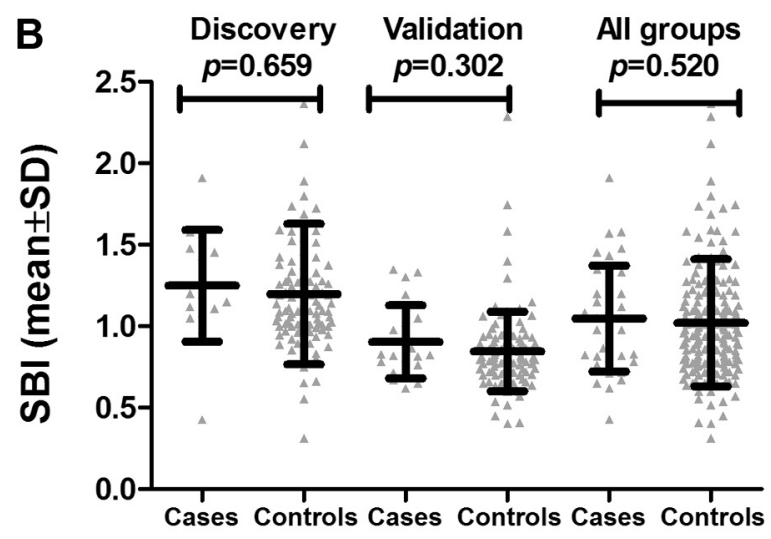

FOXP3

Figure 2. The mean differences and standard deviations of circulating IgG antibodies to annexin A1 (ANXA1) (A) and forkhead-box P3 (FOXP3) (B) between early-stage lung cancer cases and healthy controls. p-Values shown are from independent t-tests. SBI: Specific binding index.

Further analysis was performed to clarify if $\mathrm{IgG}$ antibodies to these two TAAs were significantly increased in early stages (stages I and II) of NSCLC. As shown in Table III and Figure 2, Fisher's combining probability revealed that patients with early-stage NSCLC had significantly higher levels of circulating anti-ANXA1 $\operatorname{IgG}(p=0.009)$ but not of circulating anti-FOXP3 $\operatorname{IgG}(p=0.520)$ than did controls.

\section{Discussion}

The present study replicated our initial findings that circulating IgG antibodies to ANXA1 were significantly increased in NSCLC but failed to replicate the FOXP3 finding (Tables II and III). This work supports the hypothesis that circulating $\operatorname{IgG}$ antibodies to linear peptide antigens derived from the ANXA1 protein may be suitable for the development of an early diagnostic tool for NSCLC.

The failure to replicate the FOXP3 finding may arise for many reasons, including sample power, random errors and sample quality. In our previous work, we recruited a total of 272 patients with NSCLC at the Department of Pulmonary Oncology, Third Affiliated Hospital of Harbin Medical University, Harbin, China (18) in order to detect circulating anti-FOXP3 IgG. Apparently, the sample power in the previous study was much larger than that in the present study. Although circulating anti-FOXP3 IgG levels shown in the present study had a trend toward an increase in both the discovery samples and the validation samples (Table II), this 
Table II. Levels of circulating IgG antibodies to annexin A1 (ANXA1) and forkhead-box P3 (FOXP3) in non-small cell lung cancer. Data are mean \pm SD specific binding index values.

\begin{tabular}{lcccc}
\hline Group & Patients (n) & Controls (n) & $t^{\mathrm{a}}$ & $p$-Value b \\
\hline Discovery & & & & 0.005 \\
ANXA1 & $1.10 \pm 0.25(49)$ & $0.98 \pm 0.21(108)$ & 1.51 & 0.132 \\
FOXP3 & $1.30 \pm 0.41(49)$ & $1.20 \pm 0.43(108)$ & 1.183 & 0.238 \\
Validation & $0.75 \pm 0.18(60)$ & $0.72 \pm 0.14(108)$ & 1.48 & 0.141 \\
ANXA1 & $0.90 \pm 0.26(60)$ & $0.84 \pm 0.24(108)$ & \\
FOXP3 & & & \\
\hline
\end{tabular}

aStudent's $t$-test (two-tailed). b Combining probabilities: anti-ANXA1 IgG level: chi-square=13.47, df=4, $p=0.009$; anti-FOXP3 IgG: chi-square=7.97, $\mathrm{df}=4, p=0.093$.

Table III. The levels of circulating IgG antibodies to annexin A1 (ANXA1) and forkhead-box P3 (FOXP3) in early stage non-small cell lung cancer. Data are mean $\pm S D$ specific binding index values.

\begin{tabular}{lcccc}
\hline Group & Patients (n) & Controls (n) & $t^{\text {a }}$ & $p$-Value b \\
\hline Discovery & & & 1.59 & 0.115 \\
ANXA1 & $1.09 \pm 0.30(15)$ & $0.98 \pm 0.21(108)$ & 0.44 & 0.659 \\
FOXP3 & $1.25 \pm 0.34(15)$ & $1.20 \pm 0.43(108)$ & & 0.010 \\
Validation & & & 2.61 & 0.302 \\
ANXA1 & $0.81 \pm 0.18(21)$ & $0.72 \pm 0.14(108)$ & 1.04 \\
FOXP3 & $0.91 \pm 0.23(21)$ & $0.84 \pm 0.24(108)$ & \\
\hline
\end{tabular}

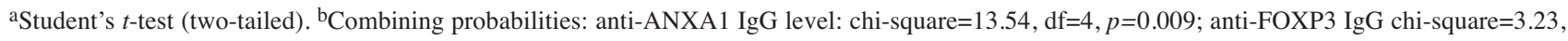
$\mathrm{df}=4, p=0.520$.

change did not reach statistical significance. Based on our initial study (18), 49 cases vs. 108 controls should have $>90 \%$ power to detect any difference in anti-FOXP3 IgG levels between the patient and control groups, suggesting that random errors of sampling may contribute to the inconsistency between these two studies.

Circulating antibodies to linear peptide antigens derived from the ANXA1 protein have a potential benefit for use in early diagnosis of NSCLC. There are more than 100 TAAs to which circulating antibodies have been analyzed in patients with cancer and the prevalence of these autoantibodies ranged from 0 to $69 \%$ (1). The linear peptide antigens derived from these TAAs are useful in developing a powerful and economical test for antibody signatures in the circulation among patients with cancer, but further investigation is needed to replicate initial findings and validate the reproducibility and precision of this technique.

\section{Conclusion}

The main aim of this study was to replicate our initial findings with different sample sets. The results support the notion that circulating IgG antibodies to linear peptide antigens from ANXA1 might be used as a biomarker for early diagnosis of NSCLC but failed to replicate our previous FOXP3 finding. Most work reported to date has focused on quantifying either mRNA or proteins in tumor tissue samples. Because antibody testing is much more simple, sensitive and reproducible as compared with quantitative real-time polymerase chain reaction and immunohistochemical analysis, it is worth carrying out further investigation to clarify whether circulating antibodies could serve as a prognostic biomarker.

\section{Acknowledgements}

The Authors thank all the patients and controls for their participation in this study. This work was supported by International collaboration project (UK and China): Developing Tumor Early Diagnosis Kit and Related Techniques.

\section{References}

1 Reuschenbach M, von Knebel Doeberitz M and Wentzensen N: A systematic review of humoral immune responses against tumor antigens. Cancer Immunol Immunother 58(10): 1535$1544,2009$. 
2 Tan HT, Low J, Lim SG and Chung MC: Serum autoantibodies as biomarkers for early cancer detection. FEBS J 276(23): 68806904, 2009.

3 Kobold S, Lutkens T, Cao Y, Bokemeyer C and Atanackovic D: Autoantibodies against tumor-related antigens: Incidence and biologic significance. Hum Immunol 71(7): 643-651, 2010.

4 Järås K and Anderson K: Autoantibodies in cancer: Prognostic biomarkers and immune activation. Expert Rev Proteomics 8(5): 577-589, 2011.

5 Liu W, Peng B, Lu Y, Xu W, Qian W and Zhang JY: Autoantibodies to tumor-associated antigens as biomarkers in cancer immunodiagnosis. Autoimmun Rev 10(6): 331-335, 2011.

6 Kobold S, Luetkens T, Cao Y, Bokemeyer C and Atanackovic D: Prognostic and diagnostic value of spontaneous tumor-related antibodies. Clin Dev Immunol 2010: 721531, 2010.

7 Piura E and Piura B: Autoantibodies to tailor-made panels of tumor-associated antigens in breast carcinoma. J Oncol 2011: 982425, 2011.

8 Piura E and Piura B: Autoantibodies to tumor-associated antigens in breast carcinoma. J Oncol 2010: 264926, 2010.

9 Zhong L, Coe SP, Stromberg AJ, Khattar NH, Jett JR and Hirschowitz EA: Profiling tumor-associated antibodies for early detection of non-small cell lung cancer. J Thorac Oncol 1(6): 513-519, 2006.

10 Chapman CJ, Murray A, McElveen JE, Sahin U, Luxemburger $\mathrm{U}$, Tureci O, Wiewrodt R, Barnes AC and Robertson JF: Autoantibodies in lung cancer: Possibilities for early detection and subsequent cure. Thorax 63(3): 228-233, 2008.

11 Jia J, Wang W, Meng W, Ding M, Ma S and Wang X: Development of a multiplex autoantibody test for detection of lung cancer. PLoS One 9(4): e95444, 2014.

12 Lam S, Boyle P, Healey GF, Maddison P, Peek L, Murray A, Chapman CJ, Allen J, Wood WC, Sewell HF and Robertson JF: EarlyCDT-lung: An immunobiomarker test as an aid to early detection of lung cancer. Cancer Prev Res (Phila) 4(7): 11261134, 2011.

13 Chapman CJ, Healey GF, Murray A, Boyle P, Robertson C, Peek LJ, Allen J, Thorpe AJ, Hamilton-Fairley G, Parsy-Kowalska CB, MacDonald IK, Jewell W, Maddison P and Robertson JF: EarlyCDT ${ }^{\circledR}$-Lung test: improved clinical utility through additional autoantibody assays. Tumour Biol 33(5): 1319-1326, 2012.
14 Jett JR, Peek LJ, Fredericks L, Jewell W, Pingleton WW and Robertson JF: Audit of the autoantibody test, EarlyCDT ${ }^{\circledR}$-lung, in 1600 patients: An evaluation of its performance in routine clinical practice. Lung Cancer 83(1): 51-55, 2014.

15 Liu L, Liu N, Liu B, Yang Y, Zhang Q, Zhang W, Yu P, Jin Y, Guo J, Guan S, Sun S, Miao L and Wei J: Are circulating autoantibodies to ABCC3 transporter a potential biomarker for lung cancer? J Cancer Res Clin Oncol 138(10): 1737-1742, 2012.

16 Ye L, Li X, Sun S, Guan S, Wang M, Guan X, Lee KH, Wei J and Liu B: A study of circulating anti-CD25 antibodies in nonsmall cell lung cancer. Clin Transl Oncol 15(8): 633-637, 2013.

17 Zhang C, Ye L, Guan S, Jin S, Wang W, Sun S, Lee KH, Wei J and Liu B: Autoantibodies against p16 protein-derived peptides may be a potential biomarker for non-small cell lung cancer. Tumour Biol 35(3): 2047-2051, 2014.

18 Wang W, Ye L, Li X, Guan S, Sun S, Wang M, Guan X, Lee K$\mathrm{H}$, Wei J and Liu B: Circulating IgG antibody against FOXP3 may be a potential biomarker for lung cancer. Advances in Lung Cancer 2(4): 79-82, 2013.

19 Wang W, Guan S, Sun S, Jin Y, Lee KH, Chen Y and Wei J: Detection of circulating antibodies to linear peptide antigens derived from ANXA1 and DDX53 in lung cancer. Tumour Biol 35(5): 4901-4905, 2014.

20 Elston RC: On fisher's method of combining $p$-values. Biometrical Journal 33(3): 339-345, 1991.
Received May 2, 2017

Revised May 14, 2017

Accepted May 16, 2017 